\title{
POTENTIAL ROLE OF VITAMIN D IN RESTORING SENSITIVITY TO mTOR INHIBITORS IN HEPATOCELLULAR CARCINOMA (HCC): 1,25(OH)VITAMIN D (VITD) REVERTS EVEROLIMUS (EVE) RESISTANCE IN A HCC CELL LINE.
}

\author{
Pivonello C. ${ }^{1}$, Provvisiero D.P. ${ }^{1}$, Negri M. ${ }^{1}$, Di Gennaro G. ${ }^{1}$, de Angelis C. ${ }^{2}$, Simeoli C. ${ }^{1}$, Galdiero G. ${ }^{1}$, De Martino M.C. ${ }^{1}$, Colao A. ${ }^{1}$, Pivonello R. ${ }^{1}$
}

${ }^{1}$ Departement of Clinical Medicine and Surgery, University of Naples «Federico II»; Naples, Italy; ${ }^{2}$ I.O.S. \& COLEMAN S.r.I., Naples, Italy

\section{BACKGROUND AND AIM}

HCC is a difficult-to-treat- cancer with poor prognosis. The recent EVOLVE-1 trial demonstrated that EVE did not improve overall survival in molecularly and clinically unselected patients with advanced HCC resistant to sorafenib treatment. In selected patients, the well-established antitumor effect of EVE could make this drug a potential adjuvant therapy. Unfortunately, the acquired resistance to this molecule due to the tumour adaptation to chronic drug use is a current challenge. VitD has been deemed as potential regimen to treat a variety of cancers alone or in combination with other drugs. The aim of this study was to assess the antiproliferative effect of the combined treatment with EVE and VitD in $\mathrm{JHH}-6$, a model of $\mathrm{HCC}$ cell line, and to explore the role of VitD pre-treatment in the re-sensitization to EVE in $\mathrm{JHH}-6$ cell line resistant to EVE (JHH-6 RR)

\section{FIGURES}
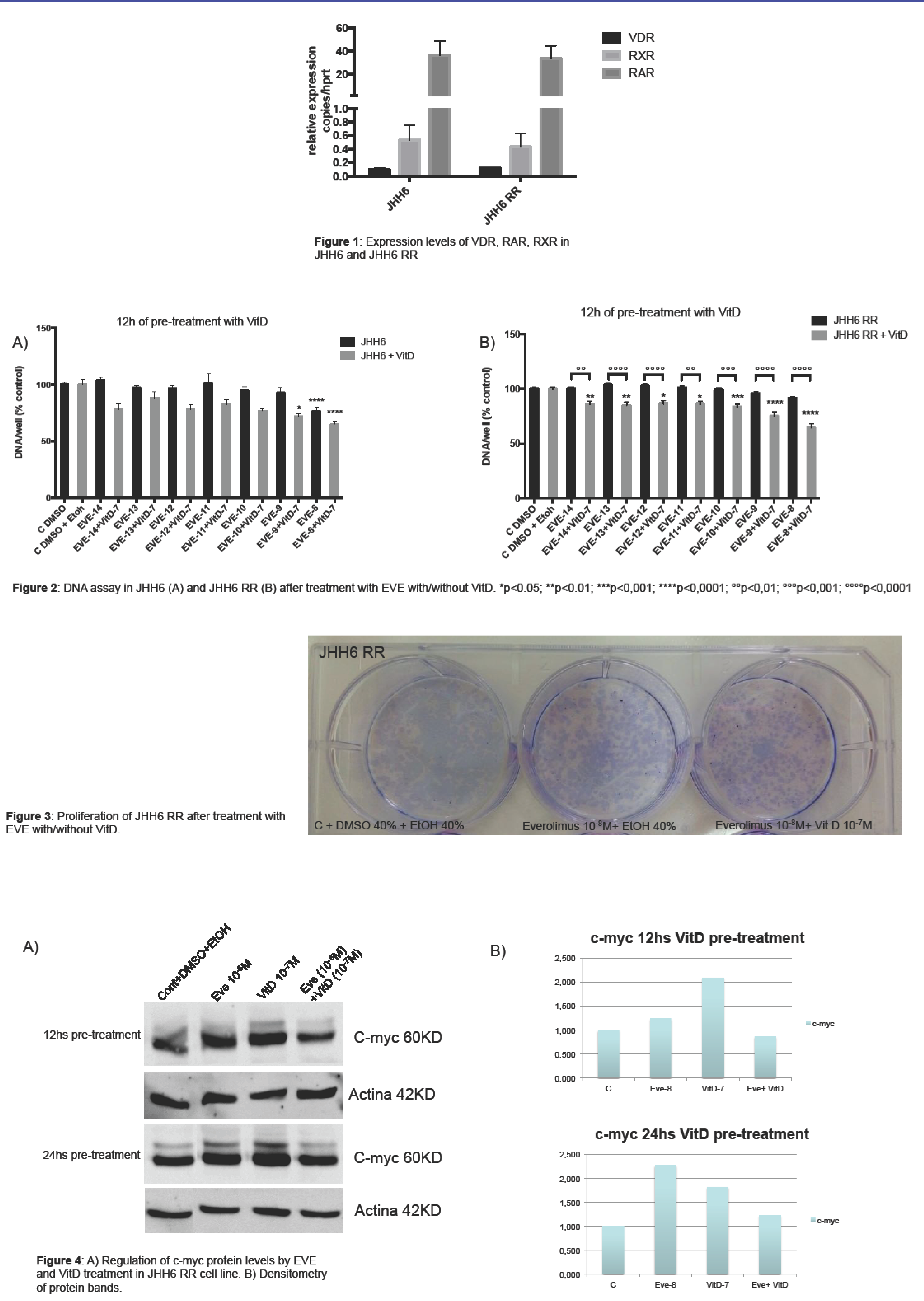

\section{METHODS}

Messenger and protein VitD receptor (VDR) expression was confirmed by RT-qPCR and immunofluorescence. To verify whether VitD can have additive effect in combination with EVE, JHH6 cell line was used. Moreover, to assess whether VitD can play a role in re-sensitization to EVE treatment, JHH-6 EVE resistant (RR) were obtained after 4 months of treatment with EVE $10^{-8} \mathrm{M}$. Cell proliferation was evaluated by measurement of total DNA content after 6 days of treatment with EVE $10^{-8} \mathrm{M}$ in $\mathrm{JHH} 6$ and in $\mathrm{JHH} 6 \mathrm{RR}$ preceded by 12 hours of pre-treatment with VitD. To better understand molecular mechanisms involved in resensitization to EVE after pre-treatment with VitD, protein levels of c-myc, a proto oncogene that is often overexpressed in many types of malignancies and known to be regulated by VitD, has been investigated.

\section{RESULTS}

JHH6 and JHH6 RR cell lines express VDR, RXR and RAR at mRNA level (Figure 1), with no difference in the expression between $\mathrm{JHH} 6$ and resistant cells. In basal condition, EVE was able to significantly reduce the proliferation index in $\mathrm{JHH} 6$ in a dose-dependent manner after 6 days of treatment and in this setting VitD did not improve EVE effect (Figure 2A). JHH-6 RR cells no longer responded to EVE treatment in concentrations from $10^{-14} \mathrm{M}$ to $10^{-8} \mathrm{M}$ (Figure $2 \mathrm{~B}$, black bars). $12 \mathrm{hs}$ of VitD pre-treatment at $10^{-7} \mathrm{M}$ was sufficient to significantly restore the efficacy of EVE at concentration ranging from $10^{-14} \mathrm{M}$ to $10^{-8} \mathrm{M}$ with a minimum effect of $14 \%$ of inhibition at $10^{-14} \mathrm{M}$ and a maximum effect of $35,3 \%$ at $10^{-8} \mathrm{M}$ $\left(10^{-14} \mathrm{M}\right.$ and $10^{-13} \mathrm{M} p<0.01$ vs Control; $10^{-12} \mathrm{M}$ and $10^{-11} \mathrm{M} p<0.05$ vs Control; $10^{-10} \mathrm{M}, 10^{-9} \mathrm{M}$ and $10^{-8} \mathrm{M} \mathrm{p}<0.001$ vs Control) as shown in Figure 3B (gray bars). In Figure 3, JHH6 RR cell growth is shown after treatment with EVE $\left(10^{-8} \mathrm{M}\right)$ with/without ViT D $\left(10^{-7} \mathrm{M}\right)$.

The analysis of c-myc protein level after drug treatment in JHH6 RR cell line revealed that after 12 and $24 \mathrm{hs}$ of VitD pretreatment, c-myc protein levels were reduced (Figure 4). Since some c-myc target genes are regulators of cell growth, it can be supposed that the reduced protein expression of c-myc is related to the VitD control growth and cellular proliferation.

\section{CONCLUSIONS}

VitD pre-treatment is able to revert the EVE resistance in JHH6 RR. The effect of VitD seems to be related to a decrease of c-myc protein expression. These preliminary data suggested the use of VitD to overcome the acquired resistance to EVE in HCC. 